ITr

يزوهشنامه مديريت حوزه آبخيز سال نهم/ شماره IV بهار و تابستان VqسI

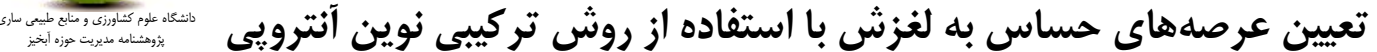

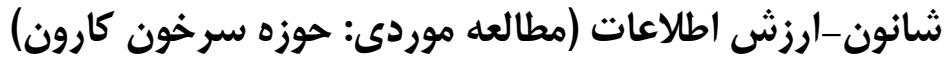

\author{
عليرضا عرب عامرى '، خليل رضايى '، كورش شيرانى 'و مجتبى يمانىع

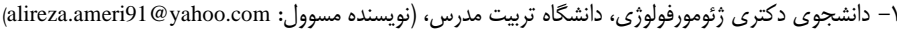

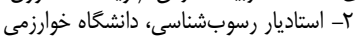

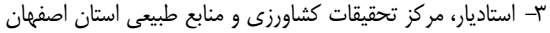

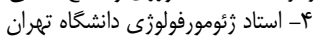

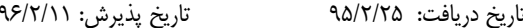

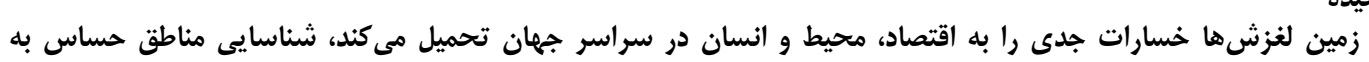

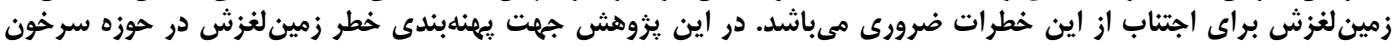

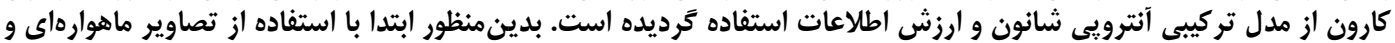

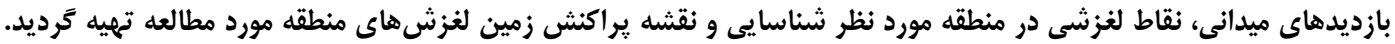

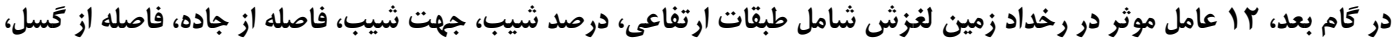

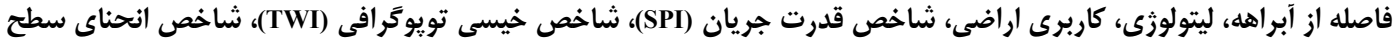

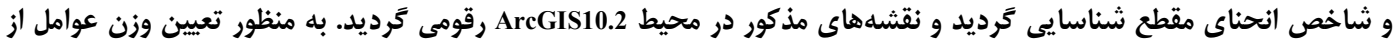

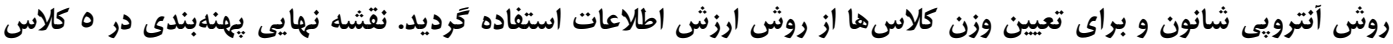

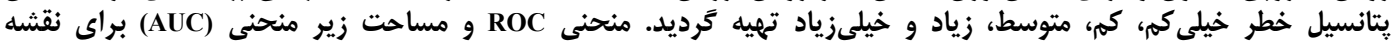

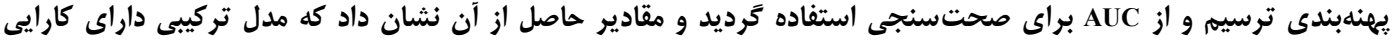

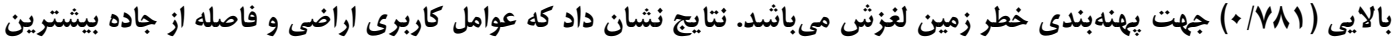

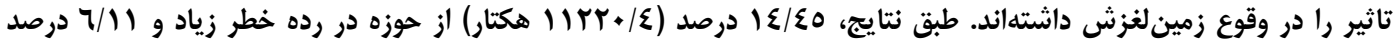

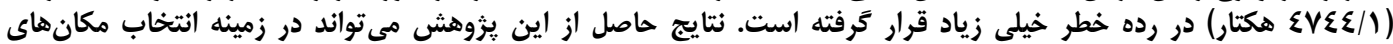

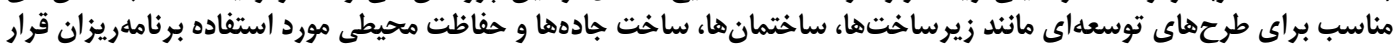

تيرد.

وازههاى كليدى: زمين لغزش، بهنه بندى، روش آنترويى شانون، روش ارزش اطلاعات، حوزه سرخون

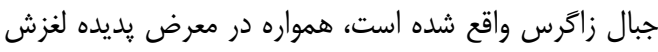

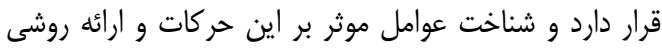

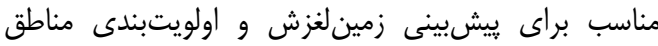

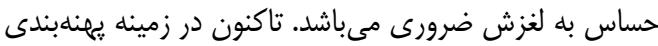

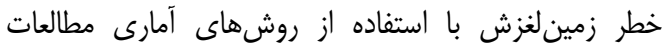

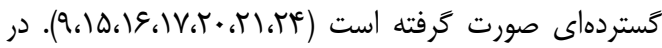

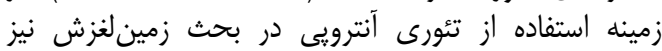

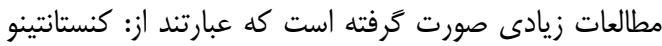

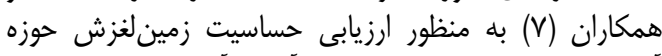

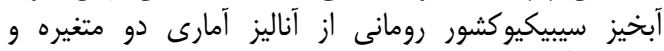
شاخص آنترويى شانون استفاده كردند. بدناريك و و همكاران آناني

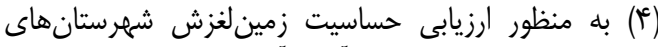

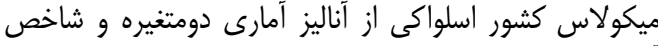

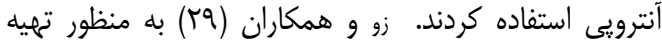

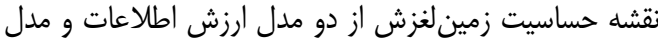

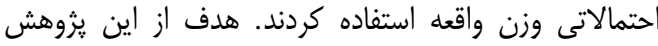

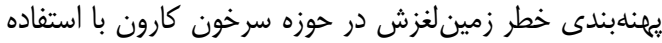

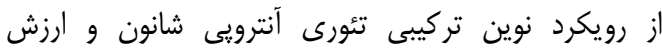

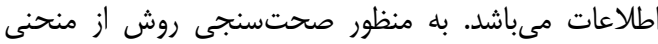
ROC استفاده شده است.

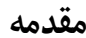

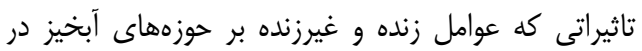

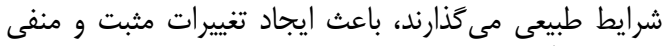

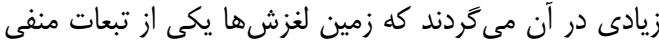

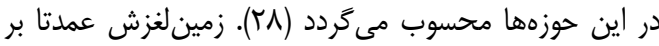

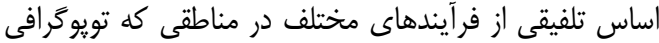

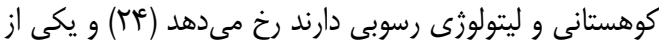

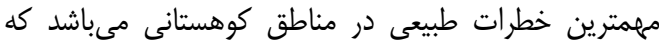

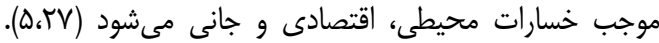

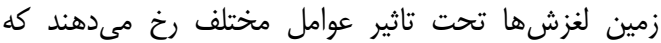

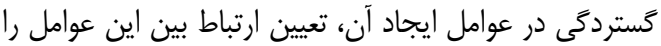

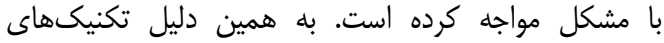

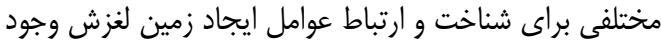

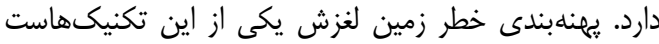

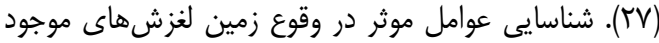

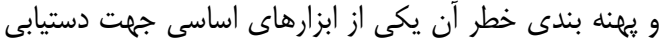

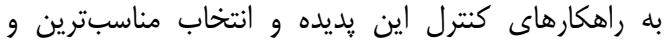

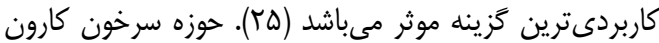

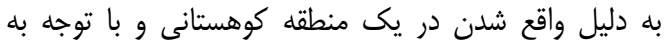
شرايط آب و هوايى و فيزيوكرافى خاص منطقه كه در سلسله 
گَندم كار و كاسه كاسه سرجشمه مئ گَيرد. رودخانه سرخون

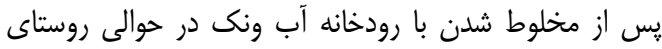

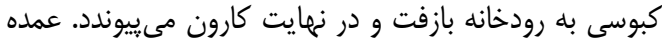

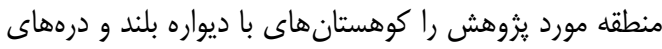

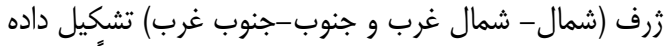

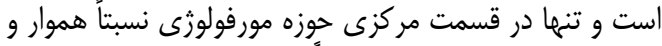

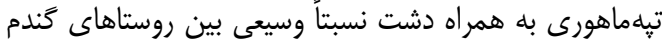

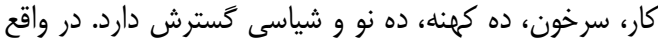

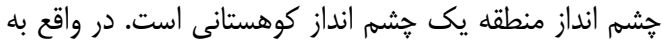

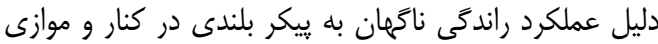

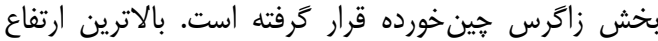

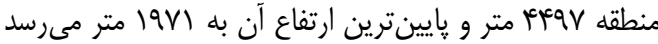

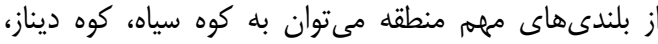

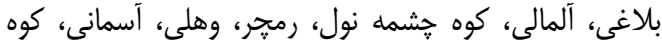
ماشكى، كوه قرش و كوه تاوه سياه اشاره كرد.

\section{مواد و روشها منطا موريت موقعيت منطقه مورد مطالعه}

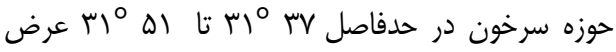

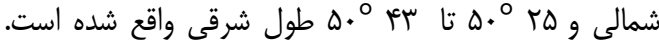

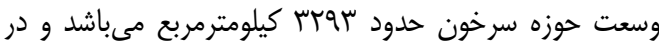
هأ كيلومترى جنوب غرب شهر شر اردل قرار دارد. اين حوزه در

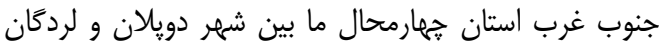

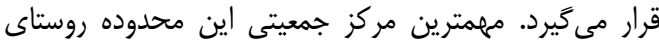

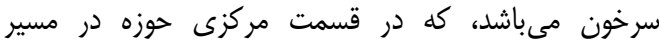

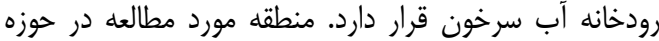

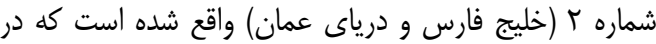

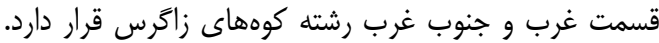

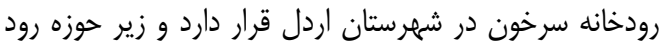

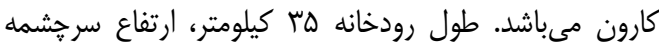

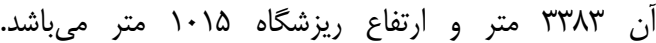
مسيررودخانه شمال غربى - جنوب شرقى است و ازت از كوههاى

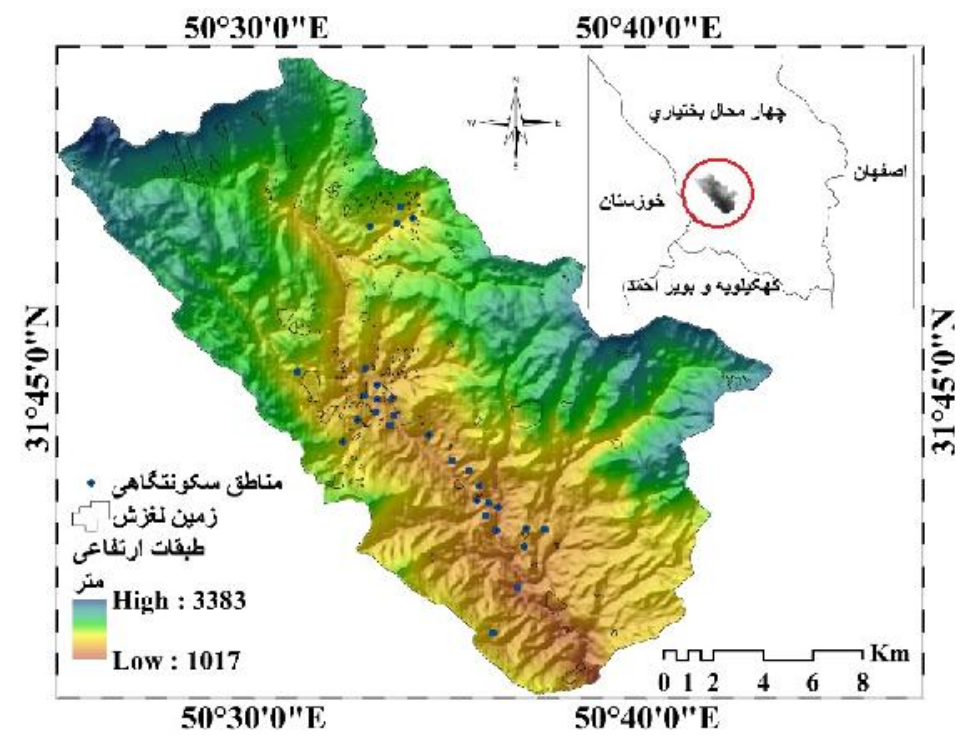

شكل 1- موقعيت منطقه مورد مطالعه

Figure 1. Location of study area

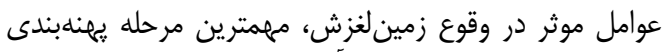

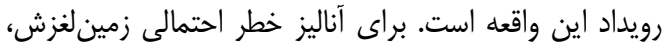

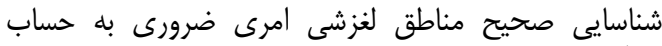

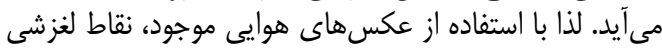

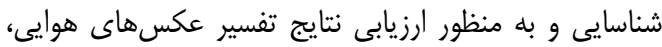

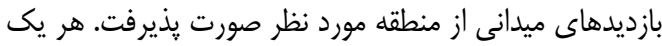

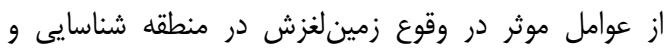
نقشههاى مربوطه در محيط نرمافزار

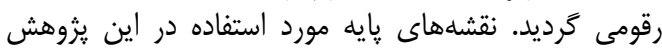

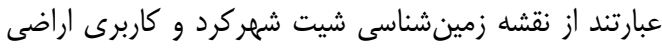

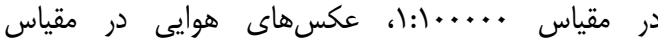

روش تحقيق اولين كام در تهيه نقشه حساسيت زمين لغزش، شناسايى

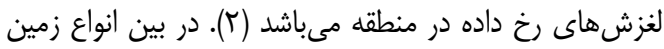

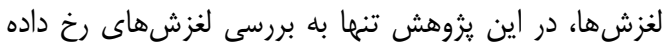

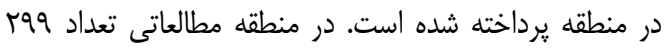

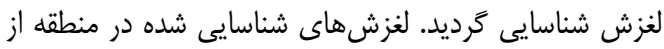

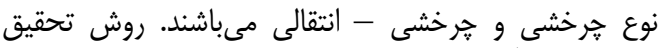

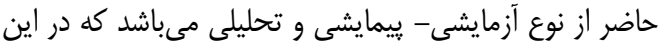

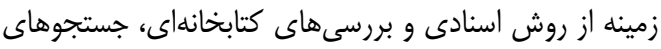

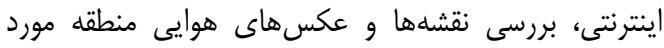
مطالعه و بررسى هاى ميدانى نيز استفاده شده است. شناسايى نقيى 
Ime

$$
\begin{aligned}
& \mathrm{G}=\frac{\mathrm{df}}{\mathrm{dx}}=\frac{\mathrm{Za}_{\mathrm{a}}+\mathrm{Z} 6+\mathrm{Z} 9-\mathrm{Z}_{1}-\mathrm{Z}_{4}-\mathrm{Z} 7}{6 \times \mathrm{P}} \\
& \mathrm{H}=\frac{\mathrm{df}}{\mathrm{dy}}=\frac{\mathrm{Z}_{1}+\mathrm{Z}_{2}+\mathrm{Z}_{\mathrm{a}}-\mathrm{Z}_{4}-\mathrm{Z}_{8}-\mathrm{Z}_{9}}{6 \times \mathrm{P}} \\
& \mathrm{p}=\frac{\mathrm{Z}_{3}+\mathrm{Z}_{6}+\mathrm{Z}_{9}-\mathrm{Z}_{1}-\mathrm{Z}_{4}-\mathrm{Z}_{7}}{6 \mathrm{~B}_{5}} \\
& \mathrm{q}=\frac{\mathrm{Z1}+\mathrm{Z}_{2}+\mathrm{Za}-\mathrm{Z} 7-\mathrm{Zg}-\mathrm{Zg}}{6 \mathrm{x} \mathbb{E}_{\mathrm{z}}} \\
& \mathrm{r}=\frac{\mathrm{Z} 1+\mathrm{Za}+\mathrm{Z} 4+\mathrm{Z} 6+\mathrm{Z} 7+\mathrm{Zg}-2 \mathrm{x}(\mathrm{Z2}+\mathrm{Z} 5+\mathrm{Zg})}{\mathrm{axd} \mathrm{I}_{\mathrm{S}}^{2}} \\
& \mathrm{~s}=\frac{-\mathrm{Z} 1+\mathrm{Za}+\mathrm{Z} 7-\mathrm{Zg}}{4 \times \Delta_{\frac{2}{2}}^{2}} \\
& \mathrm{t}=\frac{\mathrm{z1}+\mathrm{z2}+\mathrm{z} \mathrm{z}+\mathrm{z7}+\mathrm{z8}+\mathrm{z9}-2 \times(\mathrm{z} 4+\mathrm{z5}+\mathrm{z6})}{\mathrm{ax} \unlhd_{5}^{2}}
\end{aligned}
$$

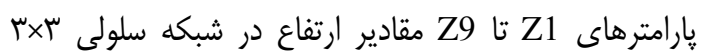

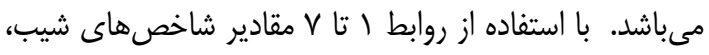
جهت شيب، انحناى سطح و انحناى مقطع محاسبه كرديد.

$$
\begin{aligned}
& \text { SLOPE }=\sqrt{\mathrm{H}^{2}+\mathrm{G}^{2}} \\
& \text { ASPECT }=\arctan \left(\frac{\mathrm{H}}{\mathrm{G}}\right)
\end{aligned}
$$$$
\text { Plan Curvature }=-\frac{q^{2} \times r-2 \times p \times q \times s+p^{2} \times t}{\left(\sqrt{1+p^{2}}+q^{2}\right)^{3}}
$$$$
\text { Profile Curvature }=-\frac{q^{2} \times r+2 \times p \times q \times r \times s+q^{2} \times t}{\left(p^{2}+q^{2}\right) \times\left(\sqrt{1+p^{2}}+q^{2}\right)^{3}}
$$$$
\text { مقادير شاخصهاى خيسى تويوگرافى و قدرت آبراهه نيز از }
$$$$
\text { طريق روابط rا و سا محاسبه خرديد. }
$$$$
T W I=\operatorname{In}\left(A_{S} / \tan \beta\right)
$$$$
S P I=A_{S} \times \tan _{\beta}
$$

كه در آن: AS: مساحت حوزه آبخيز و م: گراديان شيب بر حسب درجه مىباشد.

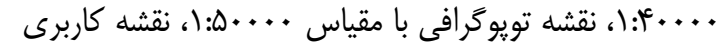

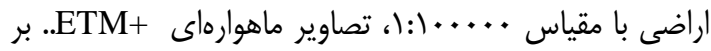

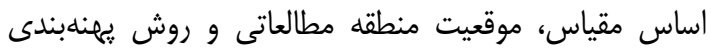

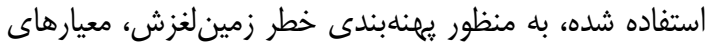

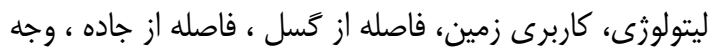

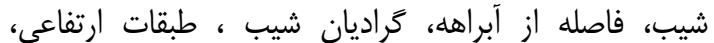

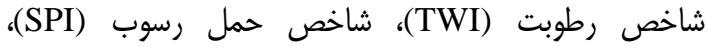

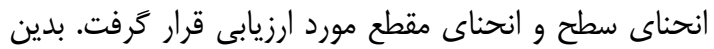

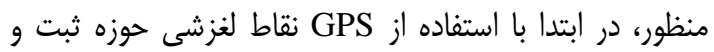

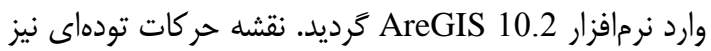
از اين طرق تهيه گرديد. در مرحله بعد با استفاده از نقشه

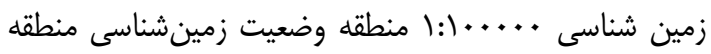

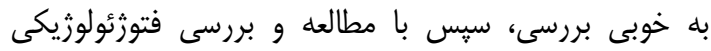

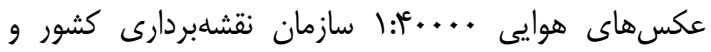

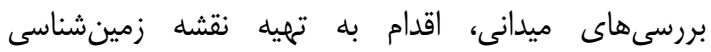

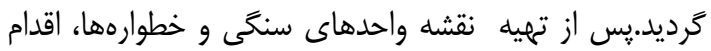

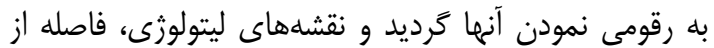

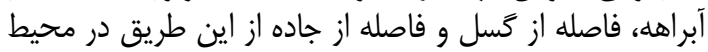
ArcGIS منطقه نيز از طريق نقشه كاربرى زمين با مقياس ل...

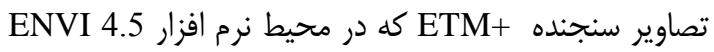

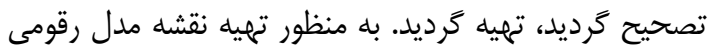

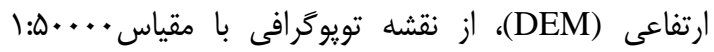

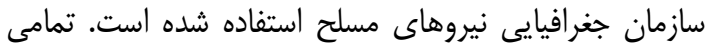

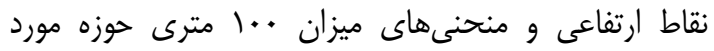

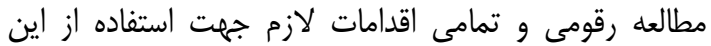

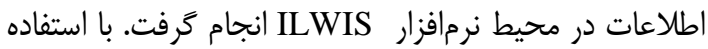

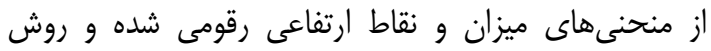

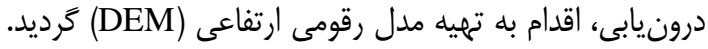

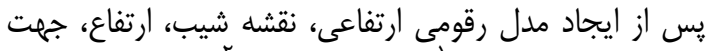

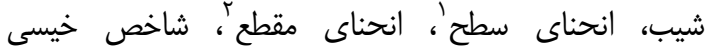

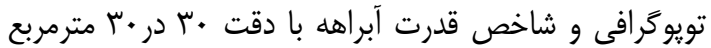

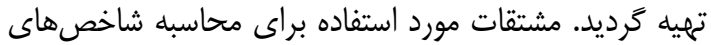
زئومورفومتريك به شردح ذيل مى باشد. 


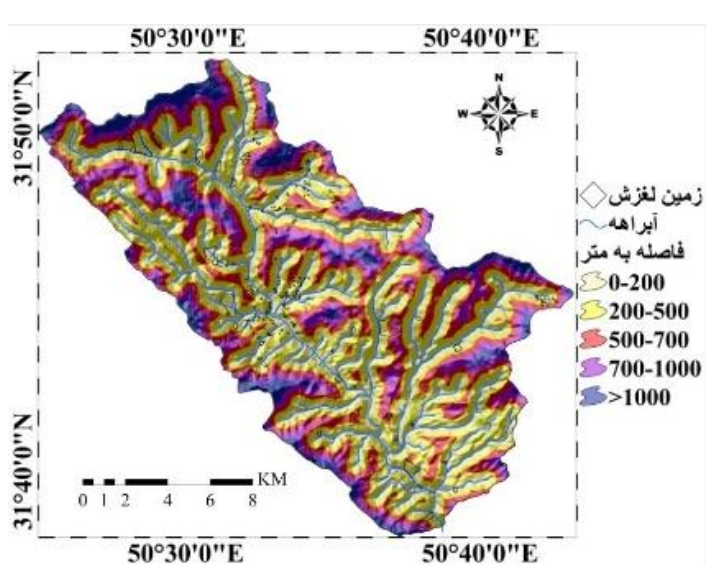

شكل م- فاصله از از آبراهه

Figure 3. Distance to stream

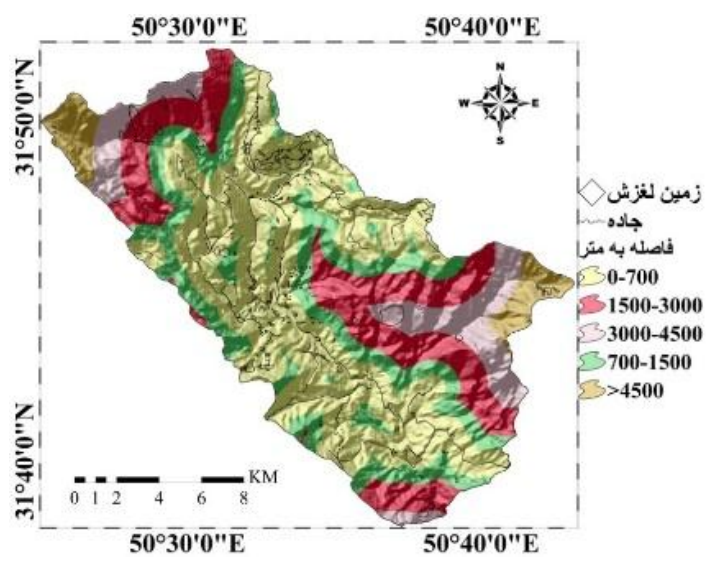

شكله - فاصله از جاده

Figure 5. Distance to road

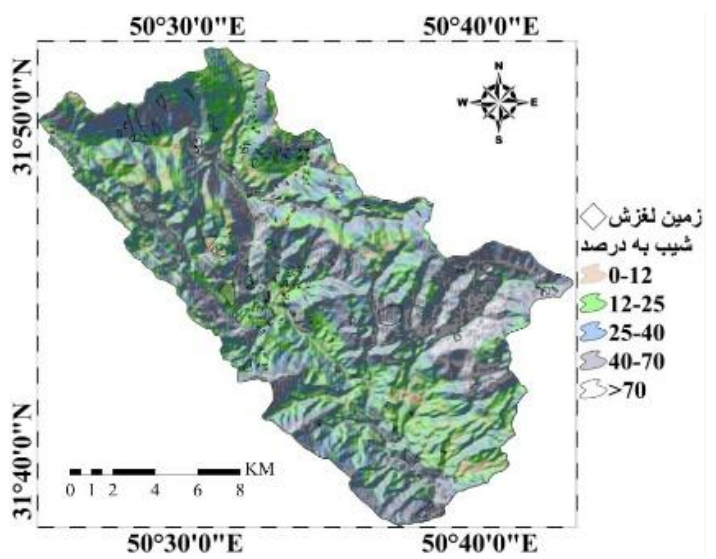

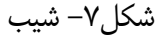

Figure 7. Slope

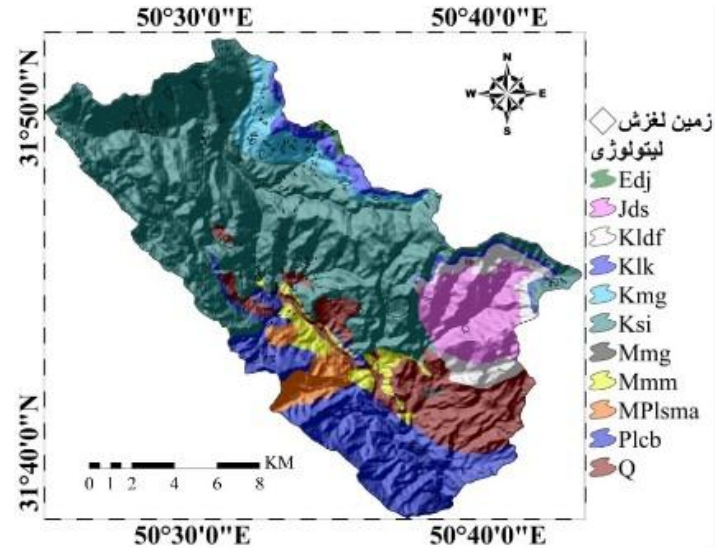

شكل ب- ليتولوزى

Figure 2. Lithology

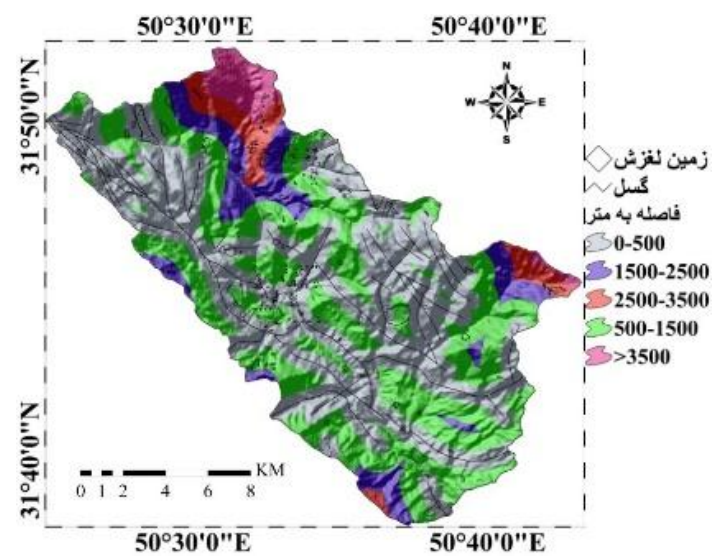

شكل أ - فاصله از خسل

Figure 4. Distance to fault

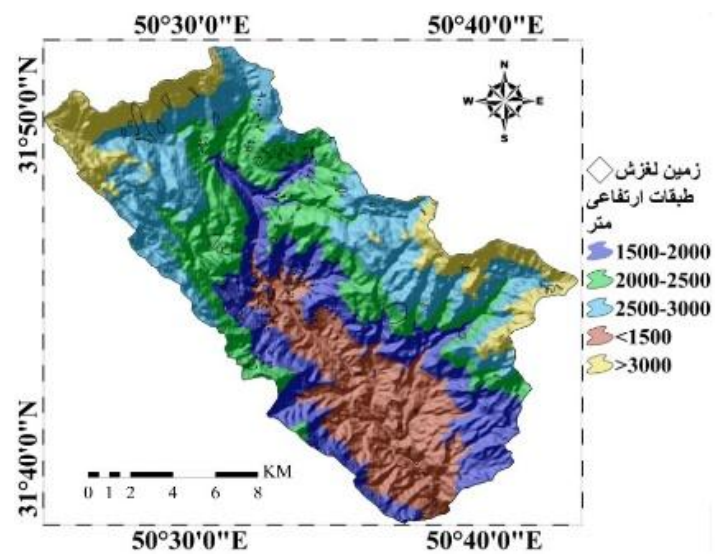

$$
\begin{aligned}
& \text { شكل }- \text { طبقات ارتفاعى } \\
& \text { Figure 6. Elevation }
\end{aligned}
$$




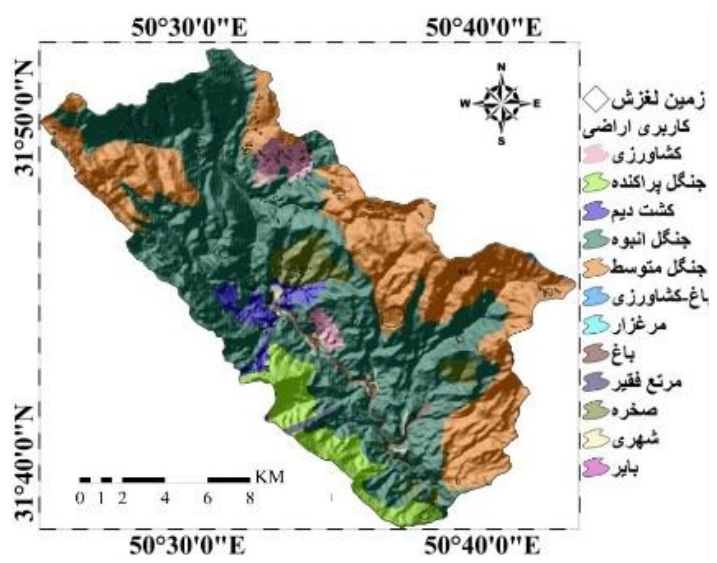

شكل 9- كاربرى اراضى

Figure 9. Land use

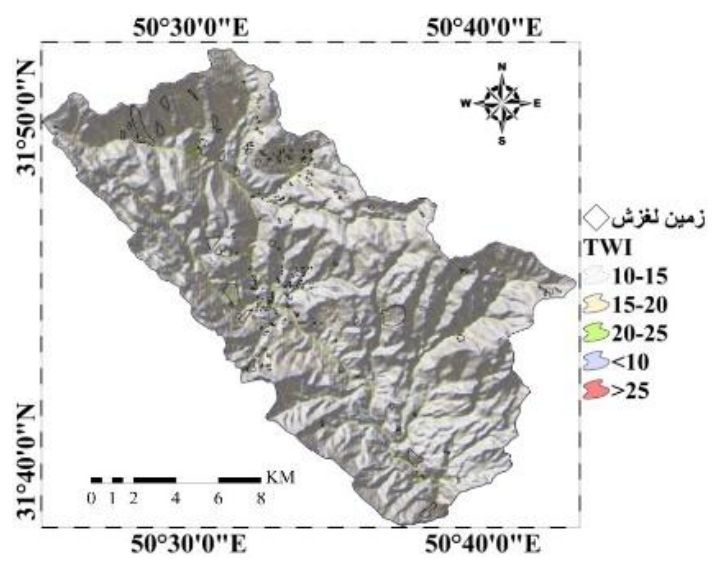

شكل |1 - شاخص خيسى تويوگرافى

Figure 11. Topography wetness index

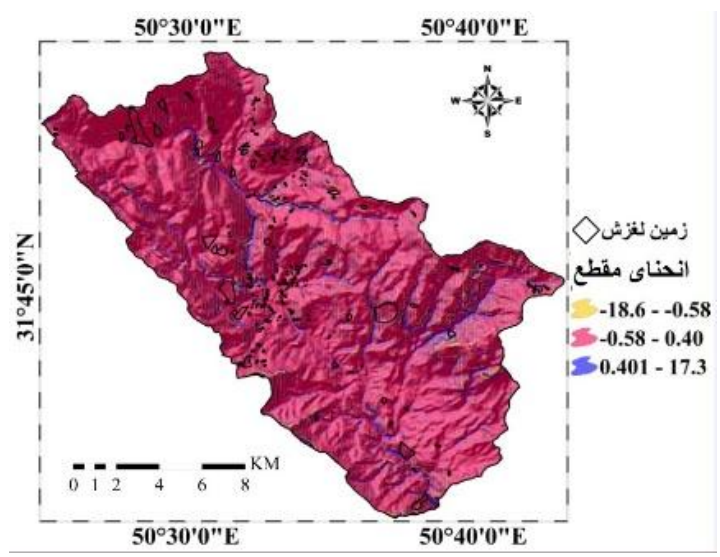

شكل سו - انحناى مقطع

Figure 13. Profile curvature

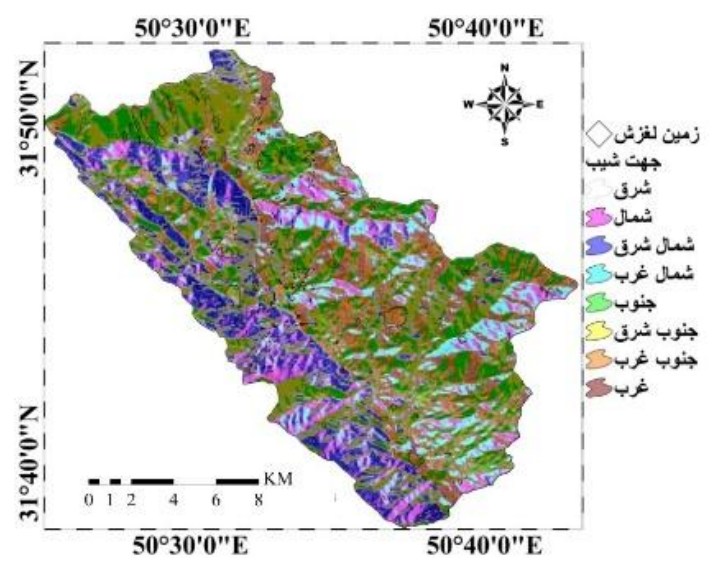

شكلی- جهت شيب

Figure 8. Slope aspect

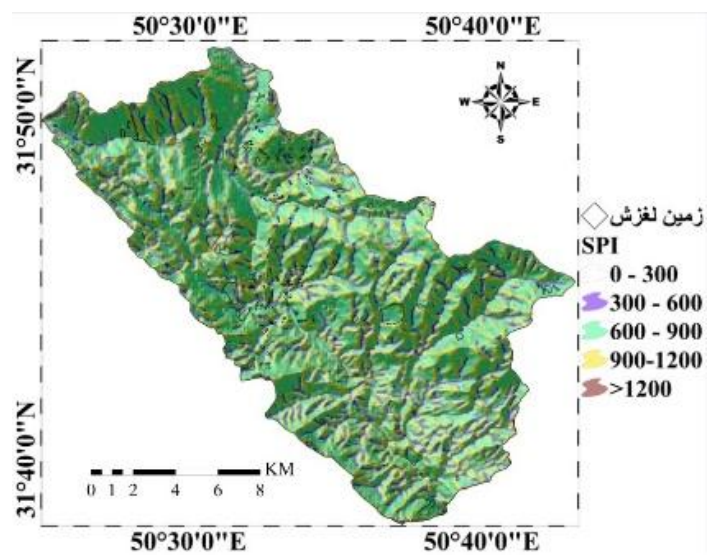

شكل • 1 - شاخص قدرت جريان

Figure 10. Stream power index

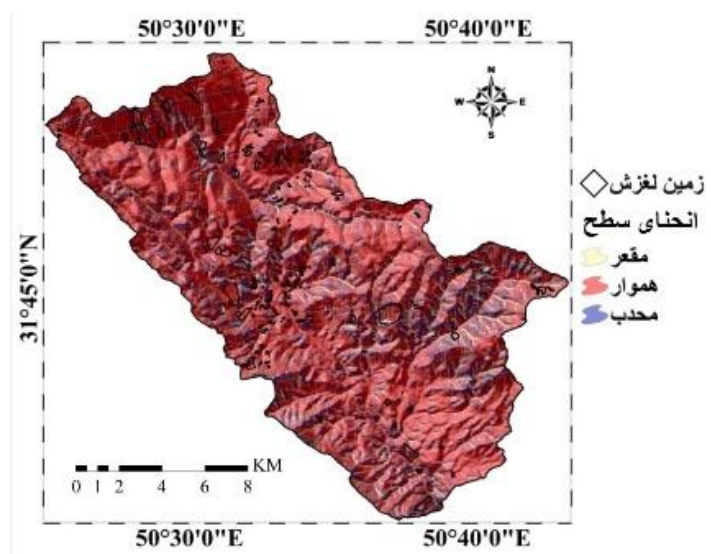

شكل rا - انحناى سطح

Figure 12. Plan curvature 
در مرحله بعد با انجام بيمايش صحرايى دقيق، موقعيت

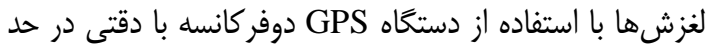

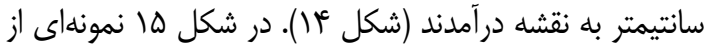

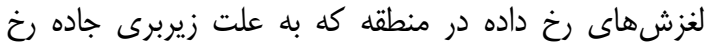
داده است، نشان داده شده است.

\section{نقشه ير اكنش زمين لغزش}

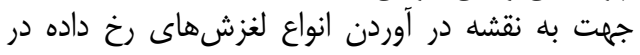

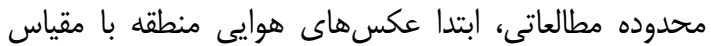

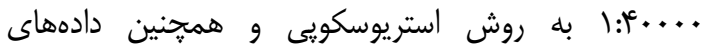

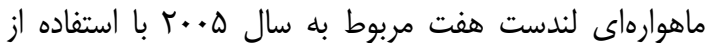
نرم افزار ENVI تفسير شدهاند و مناطق لغزشى تعيين كرديد،

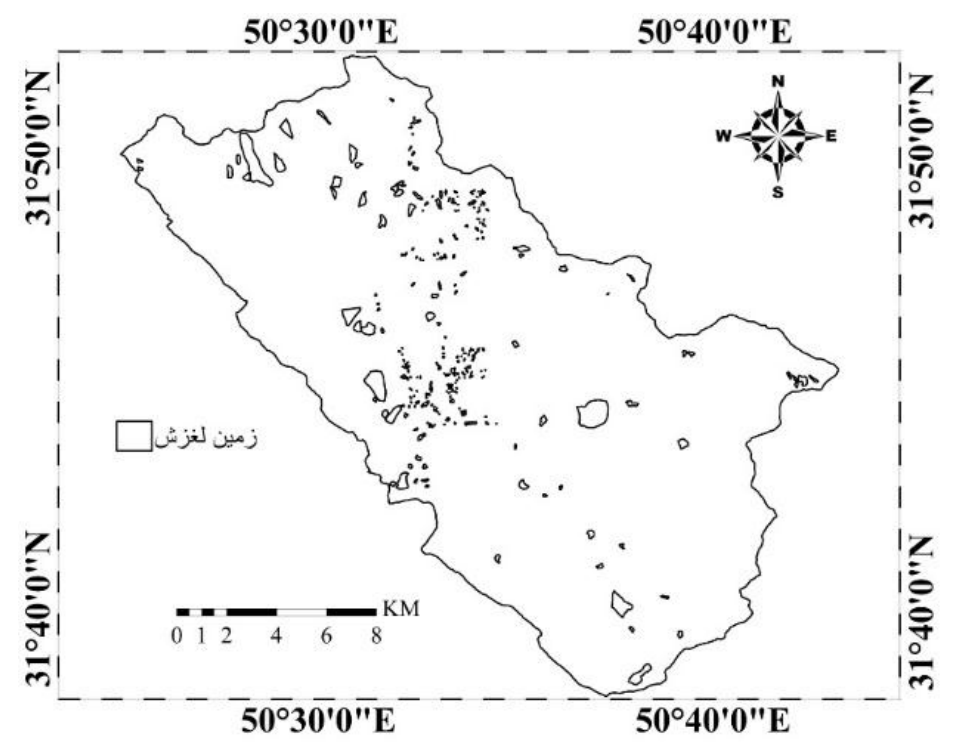

شكل عأ - نقشه براكنش زمين لغزش

Figure 14. Landslide inventory map
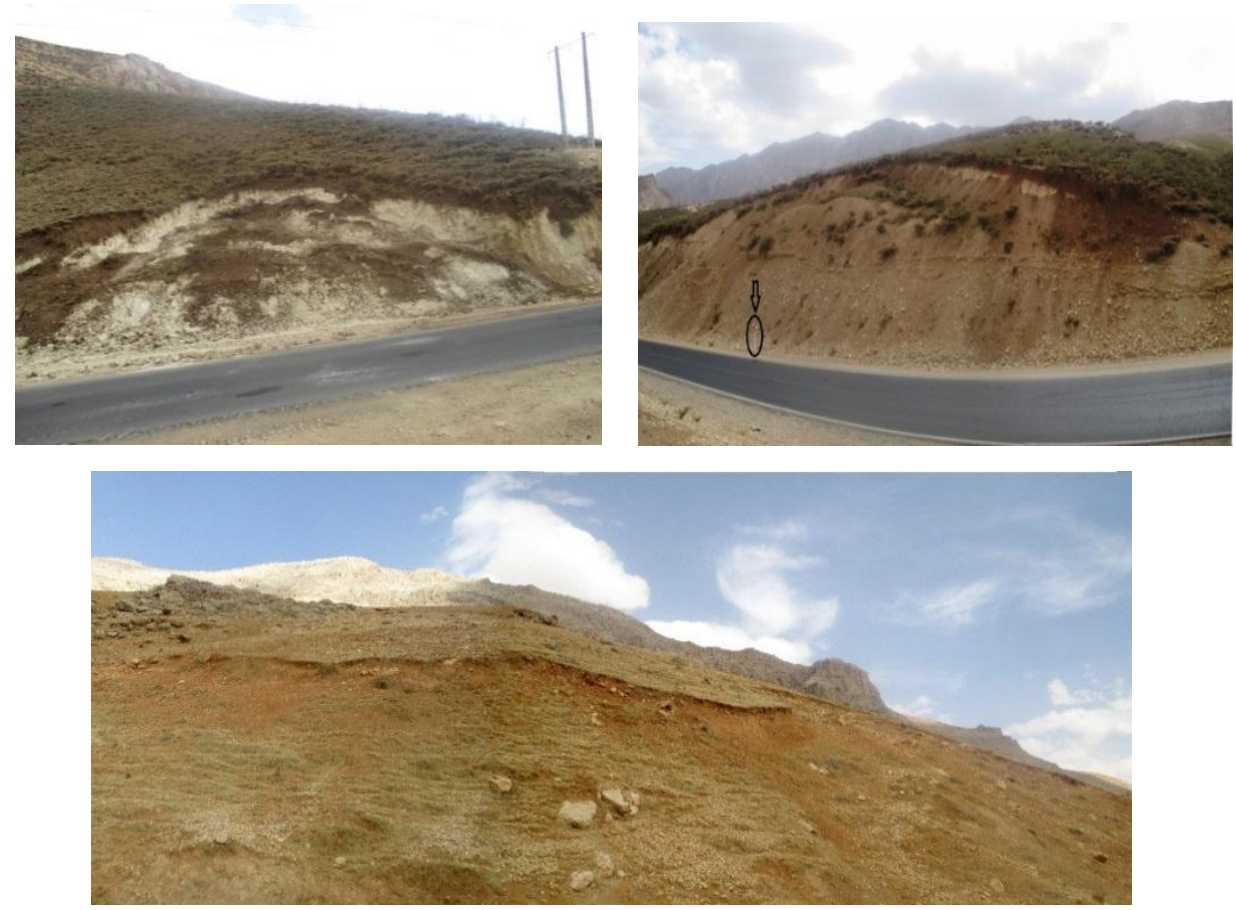

شكل 10 - نمونهاى از لغزشهاى رخ داده در منطقه

Figure 15. An example of landslides occurring in the study area 
$H_{j \max }=\log _{2} S_{j} S_{j}-$ number of classes

$\mathrm{I}_{\mathrm{j}}=\frac{\mathrm{H}_{\mathrm{j} \max -\mathrm{H}_{\mathrm{I}}}}{\mathrm{H}_{\mathrm{j} \max }}, \mathrm{I}=(0,1), \mathrm{j}=1, \ldots, \mathrm{n}$

$\mathrm{W}_{\mathrm{I}}=\mathrm{I}_{\mathrm{j}} \mathrm{P}_{\mathrm{ij}}$

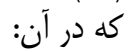

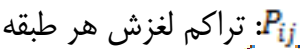

(

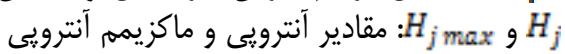
إز

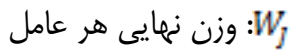

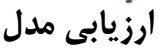

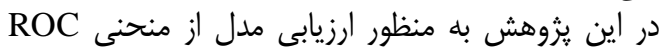

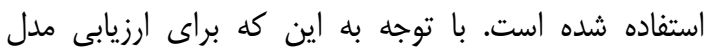

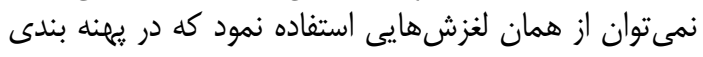

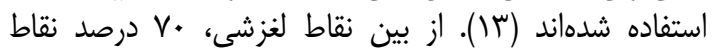

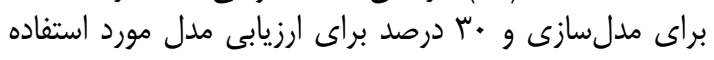

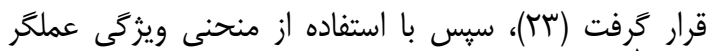

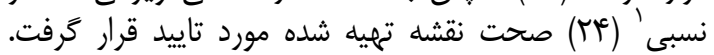

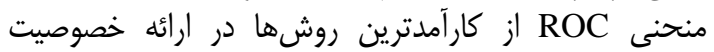

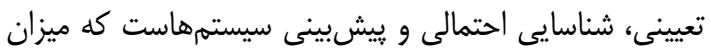
دقت مدل را به صورت كمى برآورد مى كند (1). با استفاده از

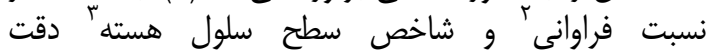

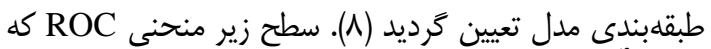

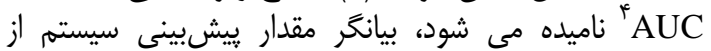

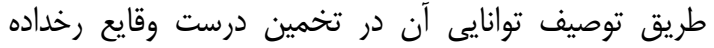

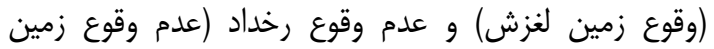

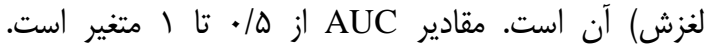

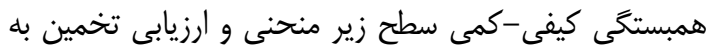

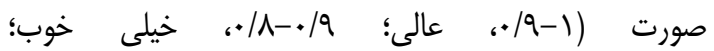

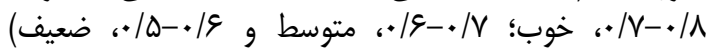

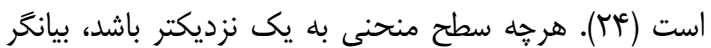

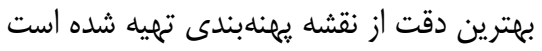

\section{نتايج و بحث}

در اين يزوهش به من منظور شناسايى و اولويتبندى عوامل

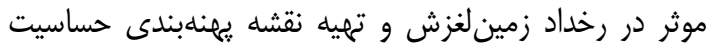

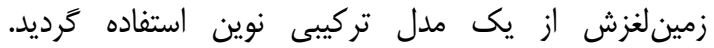
بدينصورت كه براى محاسبه وزن معيارها از روش آن آنترويى

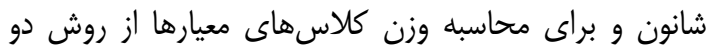

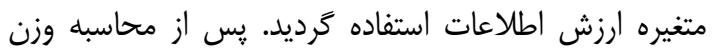

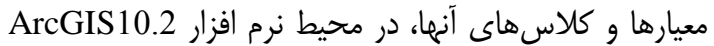

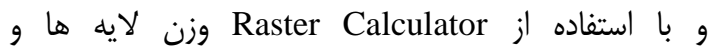

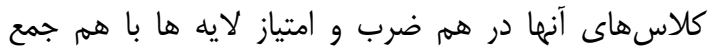

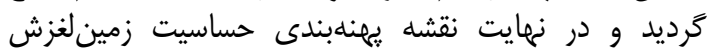

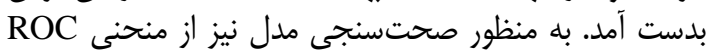

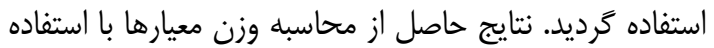

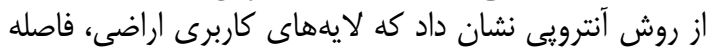

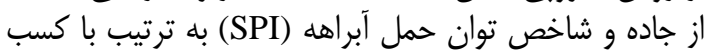

روش ارزش اطلاعات

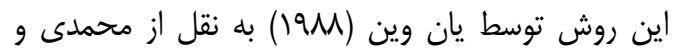

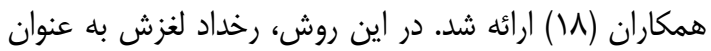

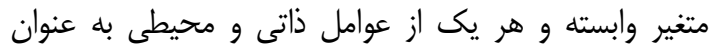

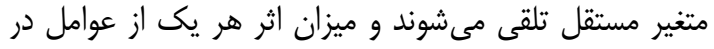

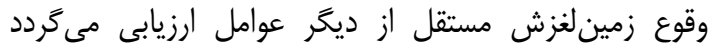

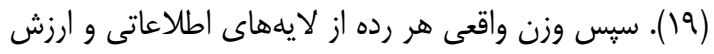

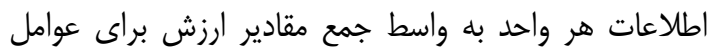

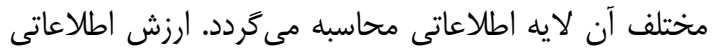

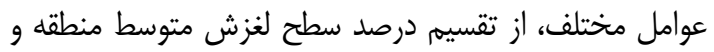

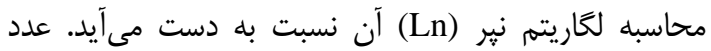

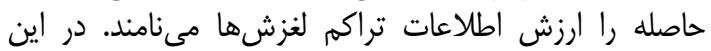

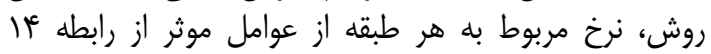

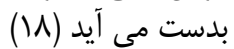

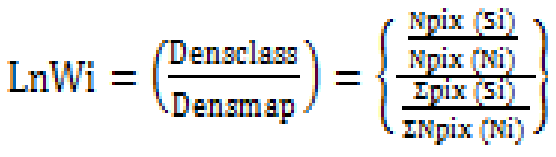

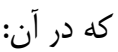

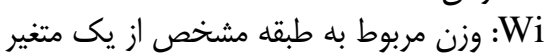

Densclass

يارامتر

Densmap

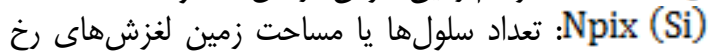
داده در هر طبقه از بارامتر

Npix (Ni)

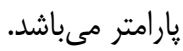
تئورى آنترويى شانون تئورى آنترويى شانون براى اولين بار تونئ توسط استفان بولتزمن

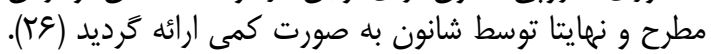

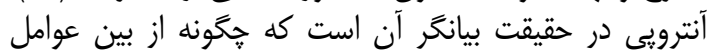

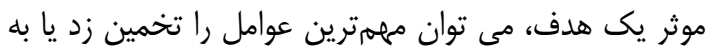

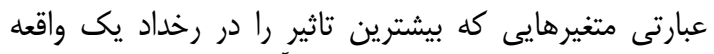

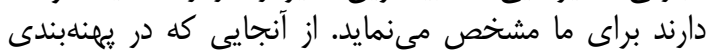

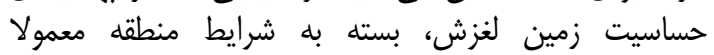

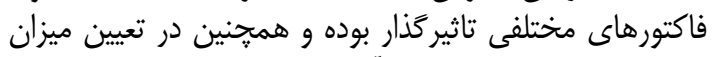

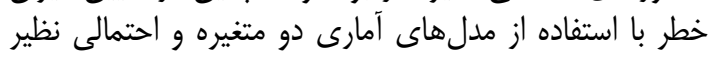

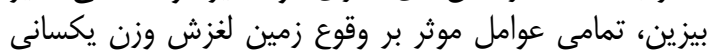

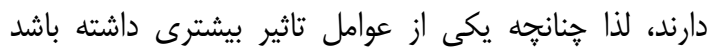

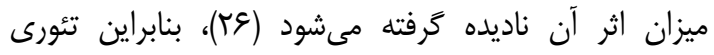

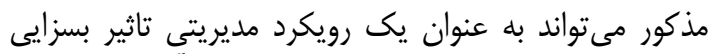

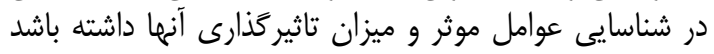

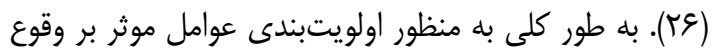

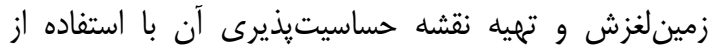

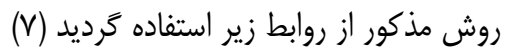

$P_{\mathrm{i} j}=\frac{a}{a}$

$\left(P_{i j}\right)=\frac{P_{i j}}{\sum_{j=1}^{S j} P_{i j}}$

$\mathrm{H}_{\mathrm{j}}=-\sum_{\mathrm{i}=1}^{\mathrm{Sj}}\left(\mathrm{P}_{\mathrm{ij}}\right) \log _{2}\left(\mathrm{P}_{\mathrm{ij}}\right)_{, j} \mathrm{j}=1_{s \ldots n} \mathrm{n}$ 
فاصله از آبراهه، ارتباط بين زمينلغزش و فاصله از اين

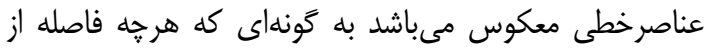

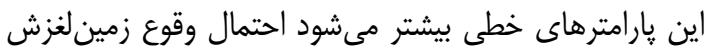

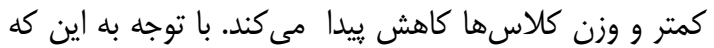

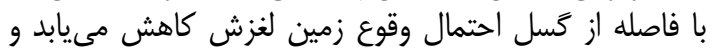

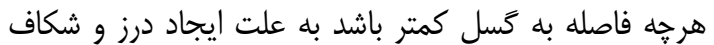

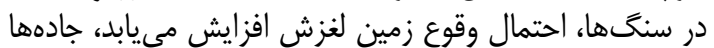

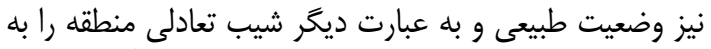

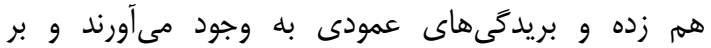

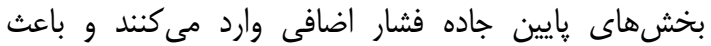

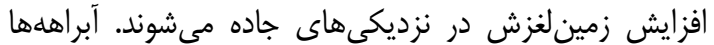
نيز با انجام زيربرى زمين

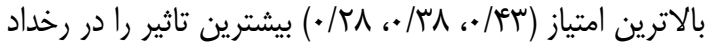

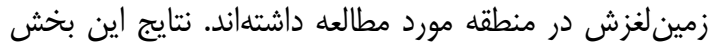

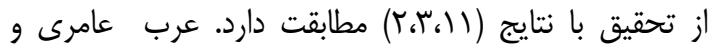

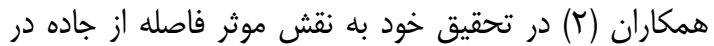

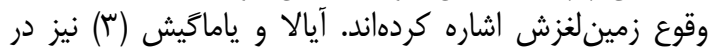

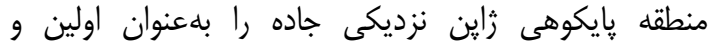

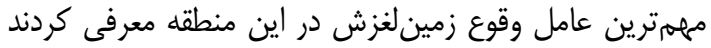

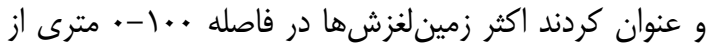

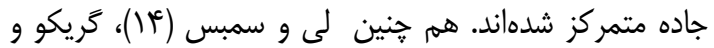

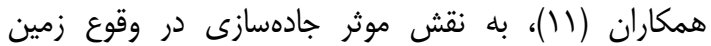

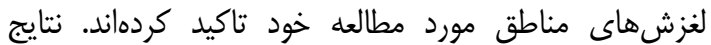

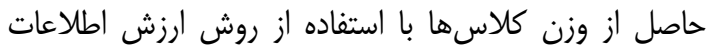

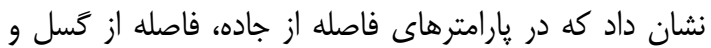

جدول ا- ارتباط بين زمين لغزشها و عوامل موثر در آن با استفاده از شاخص آنترويى Table 1. Relationship between landslides and their conditioning factors using index of entropy

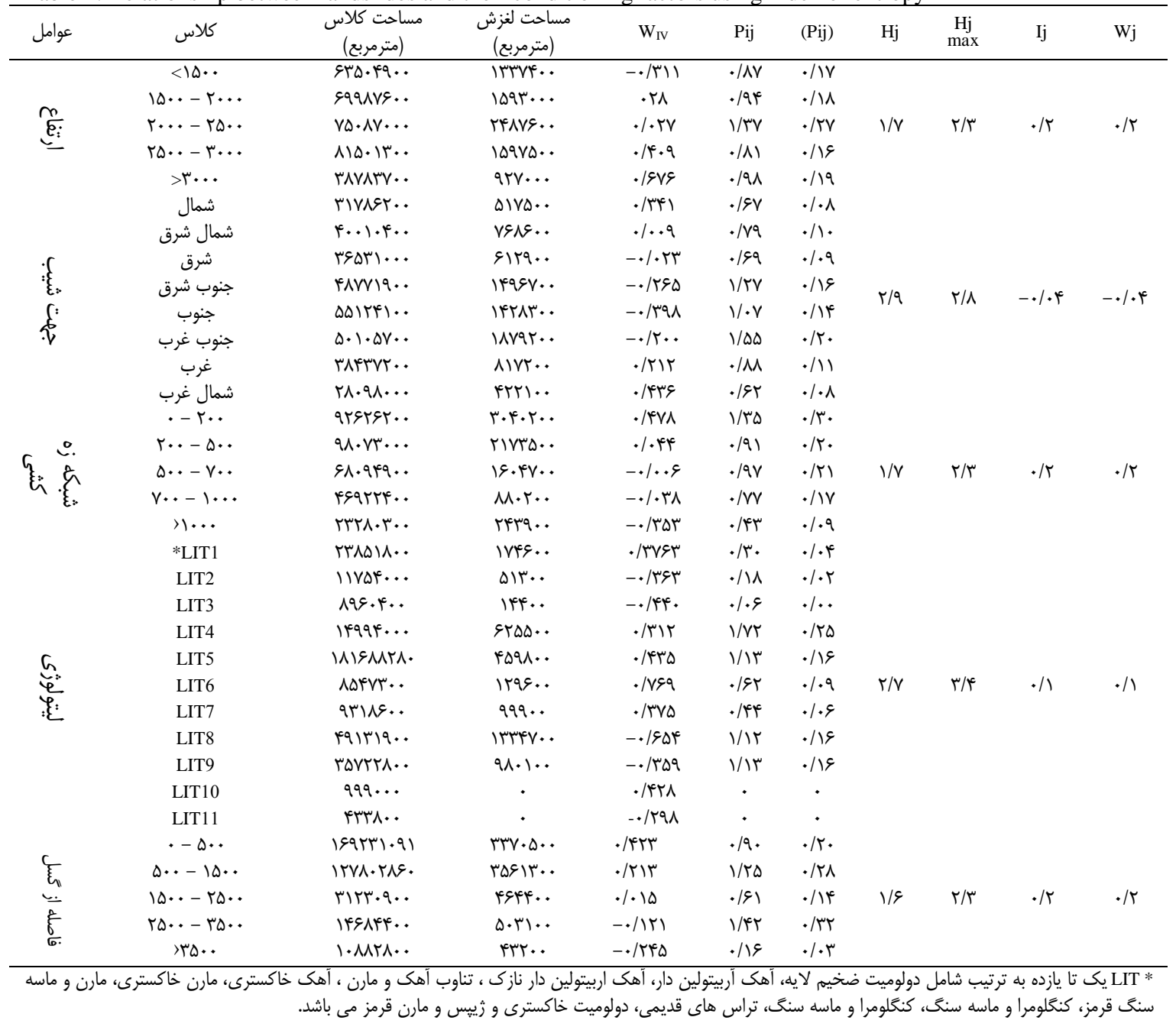



تعيين عرصههاى حساس به لغزش با استفاده از روش تركيبى نوين آنترويى شانون-ارزش اطلاعات ...

\begin{tabular}{|c|c|c|c|c|c|c|c|c|c|c|}
\hline \multicolumn{2}{|c|}{ Table 1. Continue } & \multirow[b]{2}{*}{$\begin{array}{c}\text { مساحت كلاس) } \\
\text { (مترمبع) }\end{array}$} & \multirow[b]{2}{*}{$\begin{array}{c}\text { مساحت لغز (مترمبع) } \\
\end{array}$} & \multirow[b]{2}{*}{$\mathrm{W}_{\mathrm{IV}}$} & \multirow[b]{2}{*}{ Pij } & \multirow[b]{2}{*}{$(\mathrm{Pij})$} & \multicolumn{4}{|c|}{ ادامه جدول } \\
\hline عوامل & كلاس & & & & & & $\mathrm{Hj}$ & $\begin{array}{l}\mathrm{Hj} \\
\max \end{array}$ & $\mathrm{Ij}$ & Wj \\
\hline \multirow{12}{*}{ 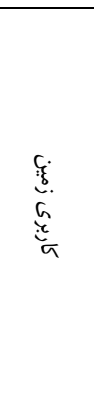 } & كشاورزى & VGISV.. & 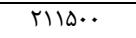 & $\cdot /$ rAV & $1 / 1 Q$ & $\cdot /$ & \multirow{13}{*}{$1 / 9$} & \multirow{13}{*}{$\Gamma / \Delta$} & \multirow{13}{*}{$\cdot / 4$} & \multirow{12}{*}{$\cdot / r$} \\
\hline & جنكل يراكنده & $19 V 4.9 .$. & TVGT.. & $\cdot / T / F$ & $\cdot / \Delta \Lambda$ & $\cdot / \cdot \Delta$ & & & & \\
\hline & كشت ديم & VএqAF.. & . . & . /TAV & s/Ar & .191 & & & & \\
\hline & جنكَل انبوه & IQTHETVNE & rer.r... & 更. &.$/ 91$ & $\cdot / \cdot 1$ & & & & \\
\hline & جنگل متوسط & १९५รTА.. & $1 \Lambda \cdot 11 .$. & .11 .9 & $\cdot / V \Delta$ & .1 .9 & & & & \\
\hline & مرغزار & Trtr.. & $\cdot$ &.$/ 4 M$ & $\cdot$ & $\cdot$ & & & & \\
\hline & باغ & rrovq.. & rra.. & $\cdot / T M$ & $\cdot / T V$ &.$/ .4$ & & & & \\
\hline & ارتفاعات باير & $9191 .$. & $\cdot$ & . /TAS & $\cdot$ & . & & & & \\
\hline & مرتع & $v_{r} \ldots$ & · & אזא/ & $\cdot$ & · & & & & \\
\hline & شهرى & IVDIf.. & $9 .$. & $\cdot$ &.$/ .4$ & $\cdot /$. & & & & \\
\hline & باغات، كشاورزى & RTAQ.. & . & ./1rq & $\cdot$ & $\cdot$ & & & & \\
\hline & صخره & IIVEIr.. & ITET.. & .1 .9 & سצ/א. & $\cdot / \cdot r$ & & & & \\
\hline \multirow{5}{*}{ 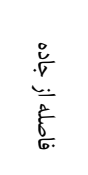 } & $\cdot-\gamma \cdot \cdot$ & IDATFOVAQ & rVYG... &. $\mid q \Delta F$ & $1 / .9$ & $\cdot|r|$ & & & & \multirow{5}{*}{$\cdot / \mu$} \\
\hline & $v \cdots-10 \cdots$ & sQTIFA.. & $v \cdot 90 .$. & TIT & . /Af & $\cdot / \cdot 1$ & \multirow{4}{*}{$1 / 9$} & \multirow{4}{*}{$r / \mu$} & \multirow{4}{*}{$\cdot / r$} & \\
\hline & $10 \cdots-r \cdots$ & qVr.rq.. & $19990 .$. & אז./. & $1 / . r$ & $\cdot / r$ & & & & \\
\hline & $r \cdots-r \Delta \cdot$. & TYESGT.. & 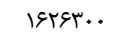 &.$- / 1 T V$ & $1 / 9 f$ & . & & & & \\
\hline & 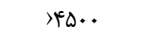 & $189.97 .$. & rift.. & & $\cdot / \Delta T$ & $\cdot 11$ & & & & \\
\hline \multirow{5}{*}{ : } & $\cdot-i t$ & rrques.. & qvr... & שTאוקו- & $1 / \pi T$ & r & \multirow{5}{*}{$1 / 1$} & \multirow{5}{*}{$r / \Gamma$} & \multirow{5}{*}{.$/ 1$} & \multirow{5}{*}{$\cdot / 1$} \\
\hline & $\pi-r \Delta$ & 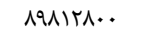 & TITVQ.. & $-\cdot / 1 \cdot r$ &.$/ 91$ &.$/ 19$ & & & & \\
\hline & $r \omega_{-}+r$. & १९৭१४৯.. & $r \cdot \Delta T \cdot \ldots$ & $\cdot / r \cdot 1$ & $\cdot / A V$ & $\cdot / 19$ & & & & \\
\hline & $f \cdot-v$. & AV.rqI... & rтrq... & - /TAV & $1 / .9$ & $\cdot / r$ & & & & \\
\hline & $>v$. & $r r \cdot q \cdot \Delta .$. & $\Delta F \wedge 11 .$. &.$/ 4 \cdot 9$ & $1 / \cdot r$ &.$/ 19$ & & & & \\
\hline \multirow{5}{*}{ 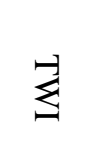 } & ». & WNৎE & rITra.. & $-\cdot / 1 \cdot r$ & $\cdot|\wedge|$ & $\cdot / \Lambda$ & \multirow{5}{*}{$1 / V$} & & & \\
\hline & $1 \cdot-10$ & ATEgV... & 1905A.. &.$- / 1 T \Delta$ &.$/ 91$ & . & & & & \\
\hline & $1 Q-r$. & IFTM.VTE & rGSAF.. & .1 .9 & $1 / 19$ & . & & $r / \mu$ & $\cdot / r$ &.$/ T$ \\
\hline & $r \cdot-r \Delta$ & $9 v 1 . f .$. & $M \ldots$. & . & $1 / 11$ & $\cdot / \pi \Delta$ & & & & \\
\hline & re & rall... & $14 .$. & $\cdot / r \cdot 1$ & . & .1 .9 & & & & \\
\hline & محدب & TAFTVGAI & NFVAFץ &.$/ 119$ & $\cdot / f F$ & $\cdot / T M I$ & & & & \\
\hline$\tilde{\xi} \frac{n}{\xi}$ & هموار & AVE\&Ta1 & ודזמאו & $-/ F \cdot T$ & $\% 1$ &.$/ . .1$ & $1 / r$ & $r / f$ & .18 &.$/ \mu$ \\
\hline & مقعر & 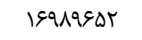 & VruVIr & .1 .91 &.$/ N T$ & תז//. & & & & \\
\hline & . r... & rтqтея.. & lIrfe.. & $-\cdot / 4 \cdot 9$ & $\cdot / f$ &.$/ \cdot r$ & & & & \\
\hline & $r \cdot .-q .$. & 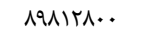 & $11 . .$. &.$- / 11$ & r & $\cdot / \cdot \wedge$ & & & & \\
\hline$\cong$ & $9 \cdots-9 \cdots$ & १९११४४.. & DFq... & .1 .9 & $r / M r$ & r & $1 / 1$ & $r / \mu$ & .10 &.$/ T$ \\
\hline & $q \cdot \cdot-\mid r \cdot$. & $\wedge \vee \cdot r q 1 \ldots$ & IDFVA.. & . $/|r|$ & $\cdot / V^{e}$ & $\cdot / r$ & & & & \\
\hline & $M \ldots+<$ & $r r \cdot q \cdot \Delta \cdot$. & G.r... &.$/ 119$ &.$/ 11$ &.$/ \cdot r$ & & & & \\
\hline & $-\mid N / \varepsilon_{-} \cdot / \Delta \Lambda$ & GDTVGTME & VGATAV & אות/. & $\cdot|r| \mid$ & $\cdot|r|$ & & & & \\
\hline$\tilde{\xi}: \frac{h}{g}$ & $-\cdot / \Delta \Lambda-\cdot / 4$ & FAVAEVAT & DFQADT & $\cdot / I V T$ & .1 .91 & $\cdot / \cdot 1$ & $1 / 1$ & $r / T$ &.$/ 4$ &.$/ T$ \\
\hline & $\cdot / f-\mid V / r$ & raAvallr & |qANTा &.$|\cdot r|$ &.$/ .14$ & $\cdot / r$ & & & & \\
\hline
\end{tabular}

است كه با نظرات (ع) مطابقت دارد. نتايج حاصل از وزن بقديه

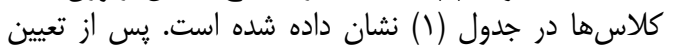

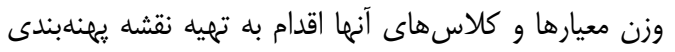

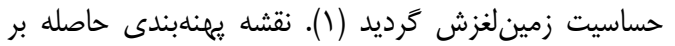

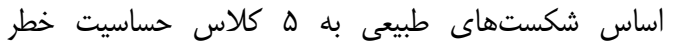

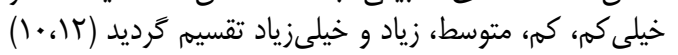

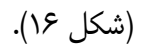

باعث نإيايدارى دامنه و ايجاد لغزش مى گرددد. يارامترهاى

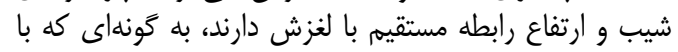

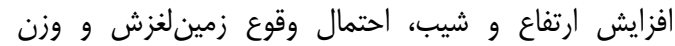

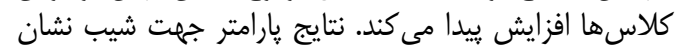

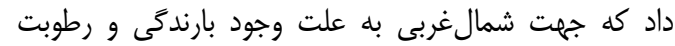

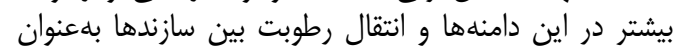

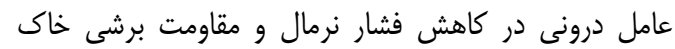




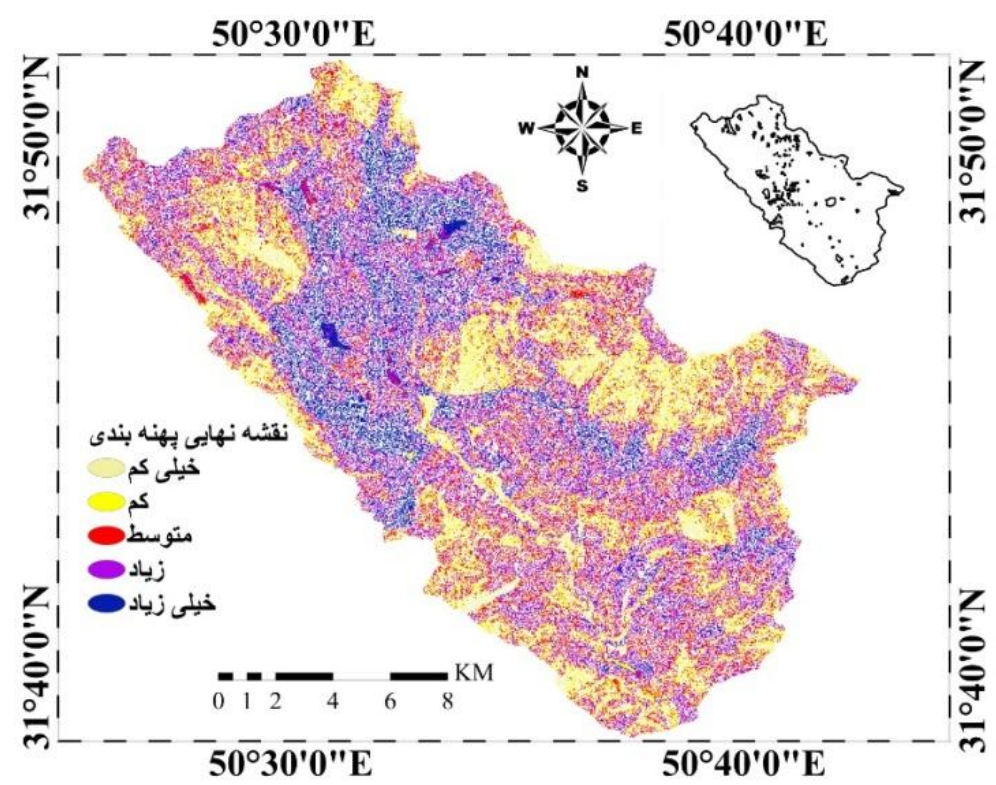

شكل عا - نقشه نمايى يهنه بندى

Figure 16. Final zonation map

مناطق لغزشى موجود و بازديدهاى ميدانى منطقه مورد مطالعه

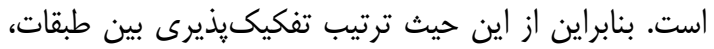

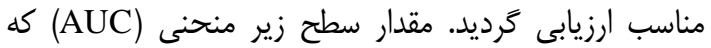

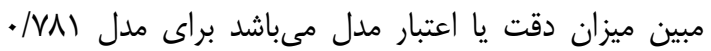

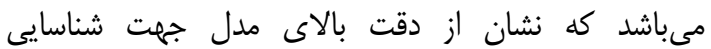

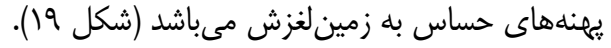

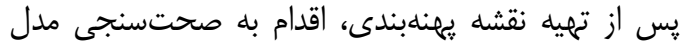

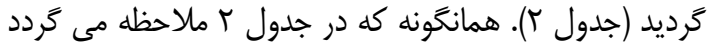

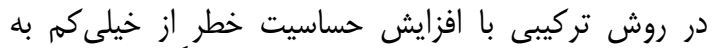

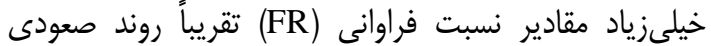

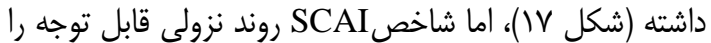

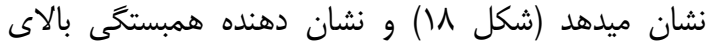
ردههاى خطر لغزش خصوصا ردههاى خيلى زياد تا زياد بان

Table 2. values of frequency ratio and seed cell area index

\begin{tabular}{|c|c|c|c|c|c|c|c|c|c|}
\hline SCAI & $\begin{array}{l}\text { درصد } \\
\text { Seed }\end{array}$ & دراوانى نسبت & درصد مساحت & مساحت هر رده خطر & لغزاحت & مساحت لغزش (مترمربع) & لغزش (مترمربع) فاقد) & خطر لغزش ردهاى & مدل \\
\hline$V / V q$ & r/ar & $.1+r$ & rr/GA & $1 V 9.9$ & $F / 9 V$ & $n \cdot 9 .$. & IVATVq... & خيلى كم & \multirow{5}{*}{ تركيبى } \\
\hline$r / 9 \Lambda$ & $11 / 11$ & $.1 \cdot 1 f$ & II & $r \Delta V \cdot q r+.$. & $r \Delta / Q$ & rqזs... & roTIle... & كم & \\
\hline I/Ar & سו & $.1 \cdot 11$ & $r \mu / \varepsilon D$ & 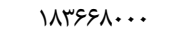 & TI/\&G & тrqq... & $1 \wedge . r s q \ldots$ & متوسط & \\
\hline . /DT & TV/M & .1 .49 & $\| f / \& \Delta$ & $\| r r+r+\ldots$ & TN/Tr & Frt... & $1 \cdot V(M) \ldots$ & زياد & \\
\hline.$/ 1 f^{c}$ & $F \Delta / 1$ & .1 .84 & $8 / 11$ & eveci... & $19 / 41$ & raDs... & REARD... & خيلى زياد & \\
\hline
\end{tabular}




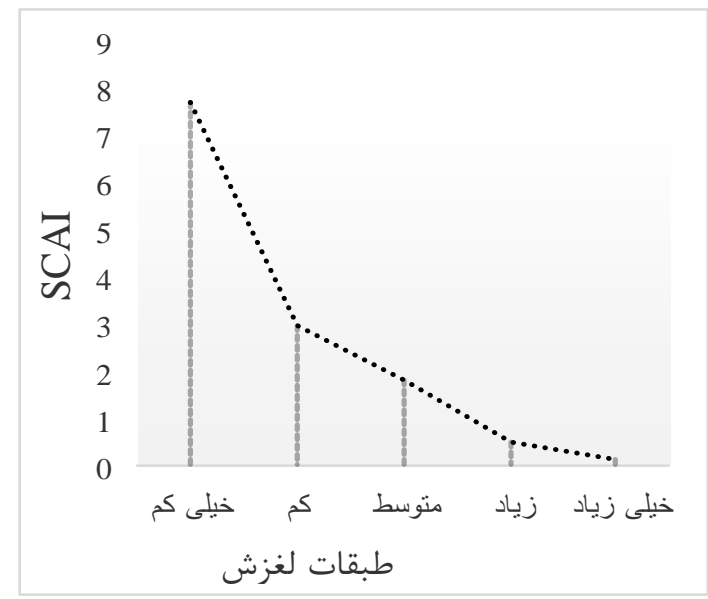

شكل 11 - روند شاخص SCAI Figure 18. Seed cell area index trend

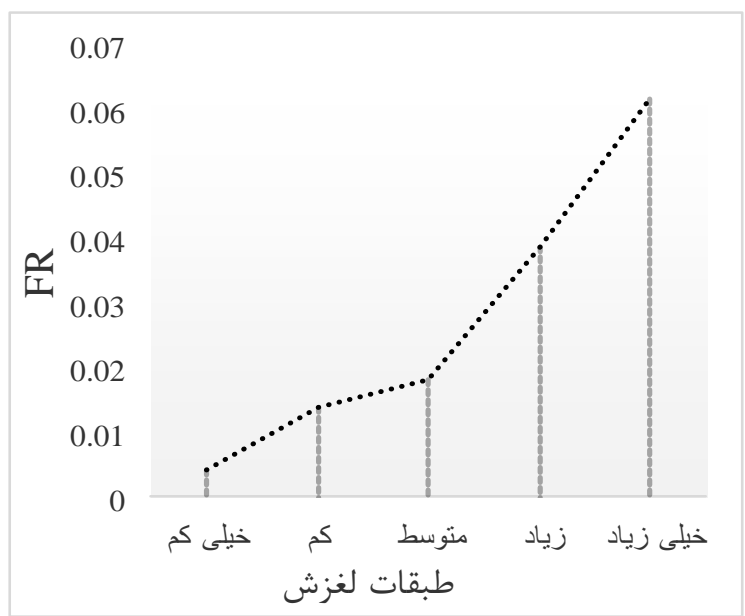

شكل IV - روند شاخص نسبت فراوانى

Figure 17. Frequency ratio trend

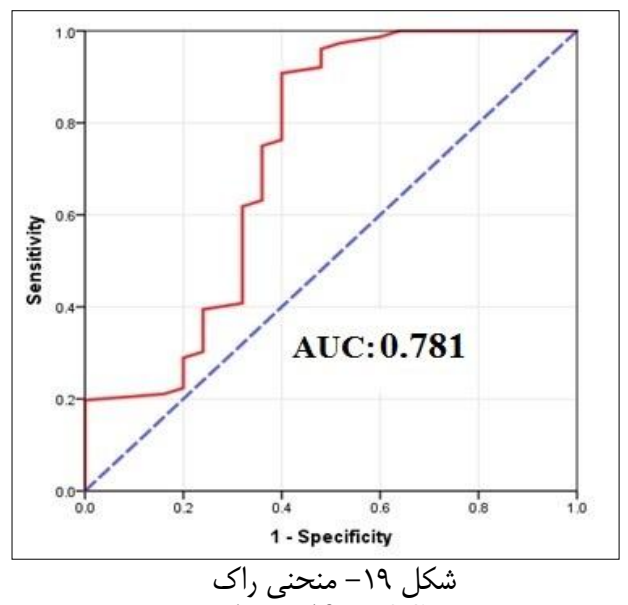

Figure 19. ROC curve

انسانى در تشديد زمين لغزش در منطقه مطالعاتى مىباشد.

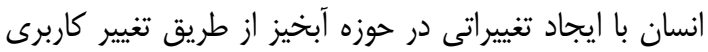

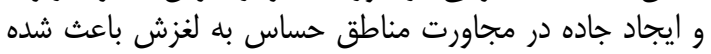

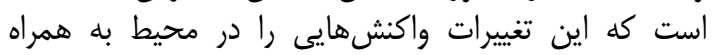

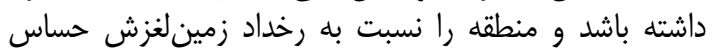

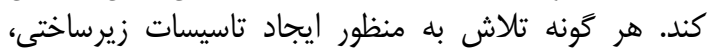

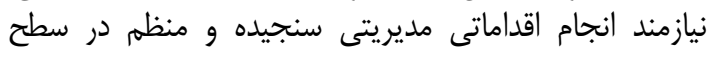

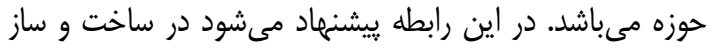

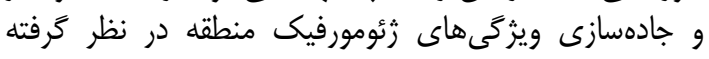

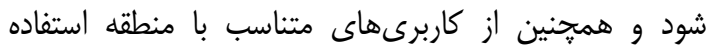

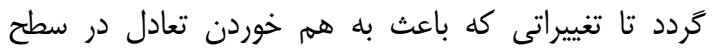

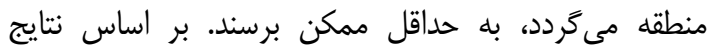

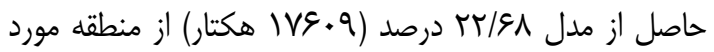

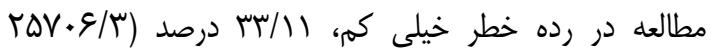

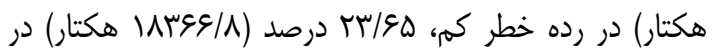

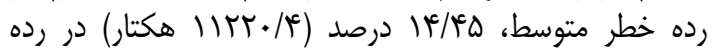

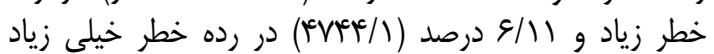
قرار گرفته است.
يديده زمين لغزش هر ساله آسيبهاى قابل توجهى را به

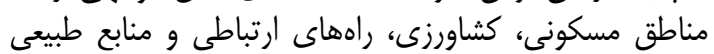

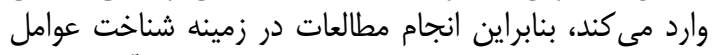

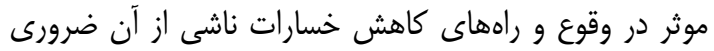

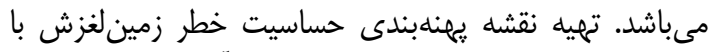

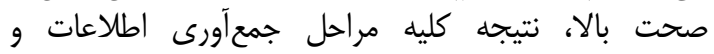

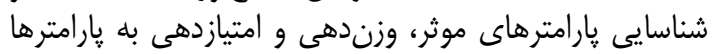

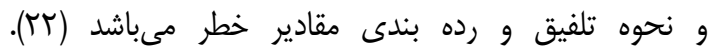

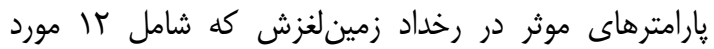

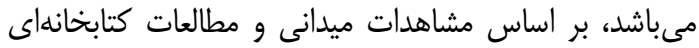

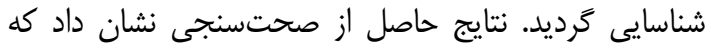

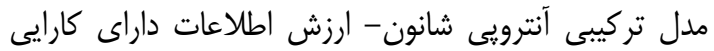

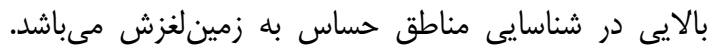

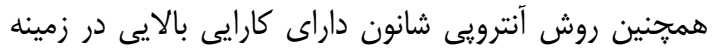

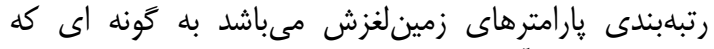

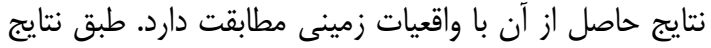

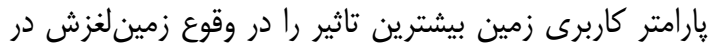
منطقه مورد مطالعه داشته است كه بيانكر تاثير فعاليتهاي 
1. Arabameri, A.R. and A.H. Halabian. 2015. Landslide Hazard Zonation Using Statistical Model of AHP (Case Study: Zarand Saveh Basin). Physical Geomorphology, 28: 65-86.

2. Arabameri, A.R. and K. Shirani. 2016. Identification of Effective Factors on Landslide Occurrence and its Hazard Zonation using Dempster-Shafer theory (Case study:Vanak Basin, Isfahan Province). Journal of Watershed Engineering and Management, 8(1): 93-106.

3. Ayalew, L. and H. Ymagishi. 2001. The application of GIS-based logistic regression for landslide susceptibility mapping in the Kakud-Yahiko Mountains, Central Japan. Geomorphology, 65: 15-31.

4. Bednarik, M., B. Magulova, M. Matys and M. Marschalko. 2010. Landslide Susceptibility Assessment of the Kral`ovany-Liptovsky' Mikulaš Railway Case Study. Physics and Chemistry of the Earth, 35: 162-171.

5. Bui, D.T., B. Pradhan, O. Lofman, I. Revhaug and O.B. Dick. 2012. Landslide susceptibility assessment in the HoaBinh province of Vietnam: A comparison of the Levenberg-Marquardt and Bayesian regularized neural networks. Geomorphology, 172: 12-29.

6. Can, T., H.A. Nefeslioglu, C. Gokceoglu, H. Sonmez and Y. Duman. 2005. Susceptibility assessments of shallow earth flows triggered by heavy rainfall at three catchment's by logistic regression analysis. Geomorphology, 82: 250-271.

7. Constantin, M., M. Bednarik, M.C. Jurchescu and M. Vlaicu. 2011. Landslide susceptibility assessment using the bivariate statistical analysis and the index of entropy in the Sibiciu Basin (Romania). Environmental Earth Science, 63: 397-406.

8. Demir, G., M. Aytekin, A. Akgun, S.B. Ikizler and O. Tatar. 2009. A comparison of landslide susceptibility mapping of the eastern part of the North Anatolian Fault Zone (Turkey) by likelihood-frequency ratio and analytic hierarchy process methods. Nat Hazards: 121-134.

9. Fatemiaghda, M., J. Ghiomian and A. Eshgheli Farahani. 2004. Evaluation efficiency statistics methods in determined Landslide hazard potential. Journal of Geosciences, 11: 28-47.

10. Guzzetti, F., A. Mondini, C. Cardinali, F. Fiorucci, M. Santangelo and K.T. Chang. 2012. Landslide inventory maps: New tools for an old problem. Earth-Science Reviews, 112: 42-66.

11. Greco, R., M. Sorriso-Valvo and E. Catalano. 2007. Logistic regression analysis in the evaluation of mass movement's susceptibility case study: Calabria. Italy. Engineering Geology, 89: 47-66.

12. Hansen, A. 1984. Engineering geomorphology: the application of an evolutionary model of Hong Kong. Zeitschrift für Gomorphologie, 51: 39-50.

13. Komac, M. 2006. A landslide susceptibility model using the Analytical Hierarchy Process method and multivariate statistics in perialpine Slovenia. Geomorphology, 74: 17-28.

14. Lee, S and T. Sambath. 2006. Landslide susceptibility mapping in the Damrei Romel area, Cambodia using frequency ratio and logistic regression models. Environmental Geology, 50: 847-855.

15. Lee, S and K. Min. 2001. Statistical analysis of landslide susceptibility at Yongin, Korea. Environmental Geology, 40: 1095-1113.

16. Maleki, A and A. Ghorbanpour. 2008. Landslide hazard zonation In Chemleh Watershed of Songhour. Journal of Geographic and Development, (12): 181-198 (In Persian).

17. Mostafai, H., M. Onagh, M. Mesdaghi and M. Shariat Jafari. 2009. Comparison between efficiency of empirical and statistical model to landslide hazard zonation (Almootrood watershed). Soil and Water Conservation Research Journal. 16: 1-16 (In Persian).

18. Mohammadi, M., H. Moradi, S. Feiznia and H. Pourghasemi. 2009. Assessment of two variate Regresion and AHP methods efficiency for Land slide hazard zoning, (A Case study: Haraz watershed). Iranian Journal of Natural Resources, 62: 539-551 (In Persian).

19. Mohammadi, M., H. Moradi, S. Feiznia and H. Pourghasemi. 2009. Ranking of effective factors on Land slide and Land slide hazard zoning maps using two variate Regresion and AHP methods, (Case study: Haraz watershed). Journal of Earth Sciences, 74: 27-32 (In Persian).

20. Mohamadi, M., H. Moradi, S. Feiznia and H. Porghasemi. 2010. Efficiency of confidence operating model, the value of information and AHP in landslide hazard zonation in Haraz watershed. Iran Natural Resources Journal, 62: 539-551. (In Persian)

21. Nasrabadi, A.H., Sh. Shatai, M. Rafatnia and M. Shariat Jafari. 2008. Assessment of efficiency to information value statistics and area density models for landslide hazard zonation. Natural Resources and Agriculture Science Journal, 15: 23-34 (In Persian).

22. Neuhauser, B. and B. Terhorst. 2006. Landslide Susceptibility Assessment Using Weights-of-Evidence Applied to a Study Area at the Jurassic Escarpment (SWGermany). Geomorphology: 1-13.

23. Pradhan, B. and S. Lee. 2010. Landslide susceptibility assessment and factor effect analysis: back propagation artificial neural networks and their comparison with frequency ratio and bivariate logistic regression modeling. Environmental Modeling and Software, 25: 747-759.

24. Pourghasemi, H.R., B. Pradhan, C. Gokceoglu and K. Deylami Moezzi. 2012. A comparative assessment of prediction capabilities of Dempster-Shafer and weights-of-evidence models in landslide susceptibility mapping using GIS.Geomatics. Natural Hazards \& Risk, 5:23-43. (in Persian)

25. Rasai, A., K. Khosravi, M. Habibnejad Roshan, A. Heidari and A. Mashayekh Khan. 2015. Lnadslide Hazard Zonation using Multivariate Regression in GIS Environment (Case Study: Aghmashhad Watershed, Mazandaran). Journal of Watershed Management Research, 6(12): 205-2015.

26. Shannon, C.E. 1948. A mathematical theory of communication. Bulletin System Technology Journal, 27: 379423.

27.Zare, M., A. Moghaddamnia, S. Tali Khoshk and H. Salmani. 2015. Landslide Hazard Assessment by using Neuro-Fuzzy Technique in VazWatershed, Journal of Watershed Management Research, 6(11): 101-110 (In Persian).

28. Zare, M., M.H. Jouri, D. Askarizadeh, T. Salarian and M. Fakhre Ghazi. 2016. An Evaluation of Landslide Hazard in Masooleh Watershed using Dempster-Shafer Theory and GIS. Journal of Watershed Management Research, 7(13): 2010-2017 (In Persian).

29. Zhu, C. and X. Wang. 2009. Landslide susceptibility mapping: A comparison of information and weights-of evidence methods in Three Gorges Area. International Conference on Environmental Science and Information Application Technology, 187: 342-346. 


\title{
Identify Areas Susceptible to Landslides using new Synthetic Method Shannon's Entropy Index-Information Value (Case Study: Karoon Sarkhon Watershed)
}

\author{
Alireza Arabameri ${ }^{1}$, Khalil Rezaei ${ }^{2}$, Kourosh Shirani ${ }^{3}$ and Mojtaba Yamani ${ }^{4}$ \\ 1- PhD Student of Geomorphology, Tarbiat Modarres University, Tehran, Iran, \\ (Corresponding Author: Alireza.ameri91@ yahoo.com) \\ 2- Assistant Professor of Sedimentology, Kharazmi University, Iran \\ 3- Assistant Professor, Isfahan Research Center for Agriculture and Natural Resources, Isfahan, Iran \\ 4- Professor of Geomorphology, Tehran University, Iran \\ Received: 14 May $2016 \quad$ Accepted: 1 May 2017
}

\begin{abstract}
The landslides impose serious damages to the economy, environment and human throughout the world. Identification of areas susceptible to landslides is necessary to avoid risks. In This research for Landslide hazard zonation in sarkhoon karoon watershed have been used Shannon's Entropy Index and Information Value Methods. For this purpose, at first, landslide locations were identified using satellite images and field surveys and then landslide inventory map was created for study area. In the next step, 10 Effective Factors in Landslide occurrence include altitude, slope, aspect, distance from road, distance from fault, distance from river, lithology, land use, stream power index, topography wetness index, Plane Curvature and Profile Curvature were identified and mentioned maps will be digitized in GIS. In order to determine the weight of factors used Shannon's Entropy Index and to determine the weight of classes used Information Value. The final Zonation map in the five classes include potential risk of very low, low, moderate, high and very high were prepared. The ROC (Receiver operating characteristic) curves and area under the curves (AUC) for landslide susceptibility map were constructed and the areas under curves were assessed for validation purpose and its value showed that the hybrid model has a higher efficiency (0.781) for landslide hazard zonation. Results showed that land use and distance to road factors have the greatest impact on landslides. According to the results of landslide maps $14.45 \%$ (11220.4 ha) of the area are ranked as very dangerous areas and $6.11 \%$ (4744.1 ha) as dangerous areas. The results of this research can help planners to choose favorable locations for development schemes, such as infrastructural, buildings, road constructions, and environmental protection.
\end{abstract}

Keywords: Information Value, Landslide, Shannon's Entropy Index, Sarkhon Watershed, Zonation 